
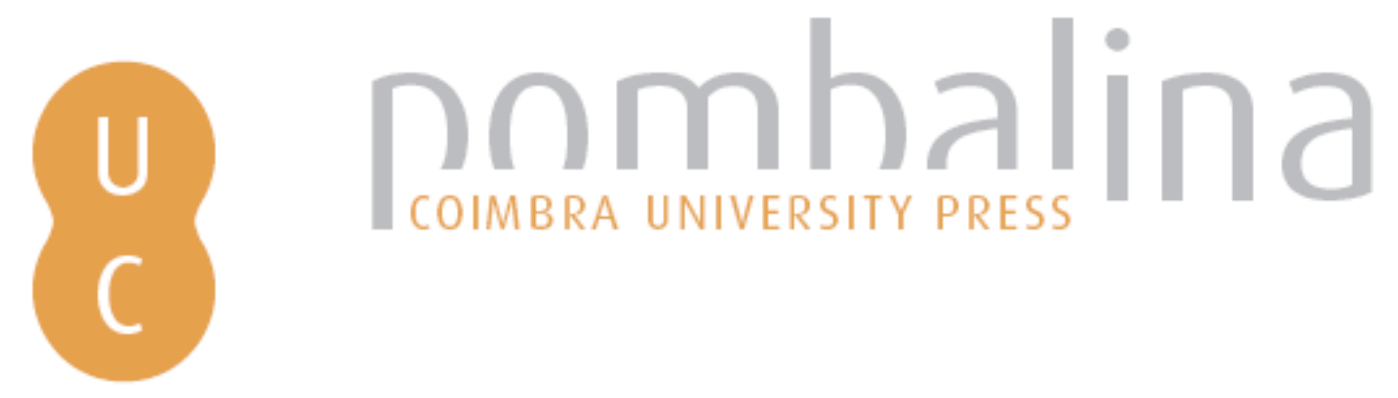

Crustáceos da Formação Codó (Bacia do Parnaíba, Brasil)

Autor(es): $\quad$ Lindoso, R. M.; Carvalho, I. S.

Publicado por: Centro de Estudos Clássicos e Humanísticos da Universidade de Coimbra

URL

persistente: URI:http://hdl.handle.net/10316.2/31419

DOI: $\quad$ DOI:http://dx.doi.org/10.14195/978-989-26-0534-0_17

Accessed : $\quad$ 26-Apr-2023 10:54:57

A navegação consulta e descarregamento dos títulos inseridos nas Bibliotecas Digitais UC Digitalis, UC Pombalina e UC Impactum, pressupõem a aceitação plena e sem reservas dos Termos e Condições de Uso destas Bibliotecas Digitais, disponíveis em https://digitalis.uc.pt/pt-pt/termos.

Conforme exposto nos referidos Termos e Condições de Uso, o descarregamento de títulos de acesso restrito requer uma licença válida de autorização devendo o utilizador aceder ao(s) documento(s) a partir de um endereço de IP da instituição detentora da supramencionada licença.

Ao utilizador é apenas permitido o descarregamento para uso pessoal, pelo que o emprego do(s) título(s) descarregado(s) para outro fim, designadamente comercial, carece de autorização do respetivo autor ou editor da obra.

Na medida em que todas as obras da UC Digitalis se encontram protegidas pelo Código do Direito de Autor e Direitos Conexos e demais legislação aplicável, toda a cópia, parcial ou total, deste documento, nos casos em que é legalmente admitida, deverá conter ou fazer-se acompanhar por este aviso.

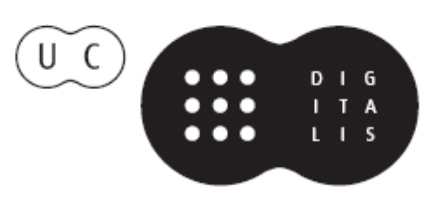





\title{
CRUSTÁCEOS DA FORMAÇÃO CODÓ (BACIA DO PARNAÍBA, BRASIL)
}

\author{
CRUSTACEANS OF CODÓ FORMATION \\ (PARNAÍBA BASIN, BRAZIL)
}

R. M. Lindoso ${ }^{1} \&$ I. S. Carvalho ${ }^{2}$

\begin{abstract}
Resumo - A Formação Codó compõe-se de arenitos, siltitos, folhelhos betuminosos e calcários em áreas descontínuas na porção central, noroeste e nordeste da Bacia do Parnaíba. Suas maiores exposiçôes são vistas em minas a céu aberto na cidade de Brejo, Estado do Maranhão. Coletas sistemáticas realizadas em dois afloramentos (Pedreira Faveirinha e Fazenda Perneta) revelaram uma rica paleobiota composta por plantas, gastrópodes, crustáceos, peixes e icnofósseis. Estes elementos da flora e fauna documentam a fase rifte sul-atlantiana. Entre os crustáceos ocorre um novo gênero e espécie de isópode Archaeoniscidae; decápodes estão representados por vários espécimes de camarões e um apêndice isolado de Brachyura. Estudos preliminares dessa paleobiota corroboram prévias inferências de ambiente lagunar com breves incursôes marinhas para a Formação Codó. Não obstante, a coleta de novos espécimes em bom estado preservacional se faz necessário para um melhor entendimento da diversidade das biotas aquáticas durante a abertura do Atlântico Sul.
\end{abstract}

Palavras-chave - Crustáceos; Formação Codó; Bacia do Parnaíba; Brasil

Abstract - The Codó Formation presents sandstones, siltstones, bituminous shales and carbonates in discontinuous areas of the central, northwest and northeastern Parnaiba Basin. The main outcrops are found in quarries of Brejo County, Maranhão State. Sampling in two outcrops (Pedreira Faveirinha and Fazenda Perneta) allowed the discovery of a wide variety of fossils such as plants, gastropods, crustaceans, fishes and ichnofossils. These specimens of the

1 Programa de Pós-Graduação em Geociências, Universidade Federal do Rio de Janeiro, Cidade Universitária - Ilha do Fundão (RJ), Brasil; rlindoso@live.com

2 Universidade Federal do Rio de Janeiro, Cidade Universitária - Ilha do Fundão, (RJ), Brasil;

ismar@geologia.ufrj.br 
flora and fauna record the beginning of the South Atlantic rift phase. Among the crustaceans there is a new genus and species of Archaeoniscidae isopod; decapods which are represented by shrimps and an isolated chelae of Brachyura. This paleobiota corroborate previous inferences concerning the occurrence of lagoon environment with marine incursions to Codó Formation. Therefore, the new data will allow a better comprehension of the aquatic biota during the South Atlantic opening in Western Gondwana.

\section{Keywords - Crustaceans; Codó Formation; Parnaíba Basin; Brazil}

\section{1 - Introduçáo}

O documentário paleontológico dos malacostracos em bacias sedimentares brasileiras é ainda incipiente, sendo representados, em sua maioria, por taxa de afinidades sistemáticas incertas. Decápodes ocorrem na Formação Marizal (Aptiano-Albiano inferior) da Bacia de Tucano, onde são encontrados camarôes da subordem Pleocyemata (Palaemon bahiensis e Atyoida roxoi) (ROXO, 1940; BEURLEN, 1950); na Formação Santana (Aptiano-Albiano) da Bacia do Araripe, na qual foram identificados camaróes paleomonídeos (Beurlenia araripensis) (MARTINS-NETO \& MEZZALIRA, 1991; SCHWEIGERT et al., 2007) e sergestídeos (Paleomattea deliciosa) (MAISEY \& CARVALHO, 1995); na Formação Gramame (Maastrichtiano) da Bacia de Pernambuco-Paraíba (MAURY, 1930; BEURLEN, 1958); na Formação Cotinguiba, Bacia de Sergipe-Alagoas, os fósseis de decápodes sáo raros e representados apenas por quelas isoladas e fragmentos de carapaças (TÁVORA \& SOUZA LIMA, 2001).

Turbay et al. (apud REIS et al., 2005) registram a ocorrência de um decápode na Formação Riachuelo, em uma localidade informalmente conhecida como Pedreira Brejo.

Isópodes são ainda mais restritos em bacias sedimentares brasileiras, com a ocorrência de apenas duas espécies: Unusuropode castroi (Turoniano do Grupo Apodi, Arenito Açu, Ceará) (DUARTE \& SANTOS, 1962) e Saucrolus silvai (Aptiano da Formação Areado, Bacia Sanfranciscana) (SANTOS, 1971).

Na Formação Codó (Aptiano da Bacia do Parnaíba), os crustáceos eram conhecidos apenas por conchostráceos e ostracodes (CARDOSO, 1962; LEITE et al., 1975; LIMA \& LEITE, 1978; KRÖMMELBEIN \& WEBER, 1985; SILVA et al., 1985, 1989). No município de Brejo, Estado do Maranhão, depósitos carbonáticos atribuídos à Formação Codó são comuns em minas a céu aberto (Fig. 1). Coletas sistemáticas realizadas em dois afloramentos (Pedreira Faveirinha e Fazenda Perneta) revelaram uma rica biota, a qual inclui fósseis de plantas, gastrópodes, crustáceos, peixes e icnofósseis (LINDOSO et al., 2011). Crustáceos decápodes são numerosos, mas mal preservados, enquanto isópodes constituem formas raras e documentam novo gênero e espécie de Archaeoniscidae para a Formação Codó.

O presente trabalho objetiva a descrição de novos crustáceos para a Formação Codó, incorporando novos taxa aqueles já existentes, possibilitando um melhor entendimento de antigas biotas aquáticas durante o estágio inicial de separação entre América do Sul e África no Gondwana Ocidental. 


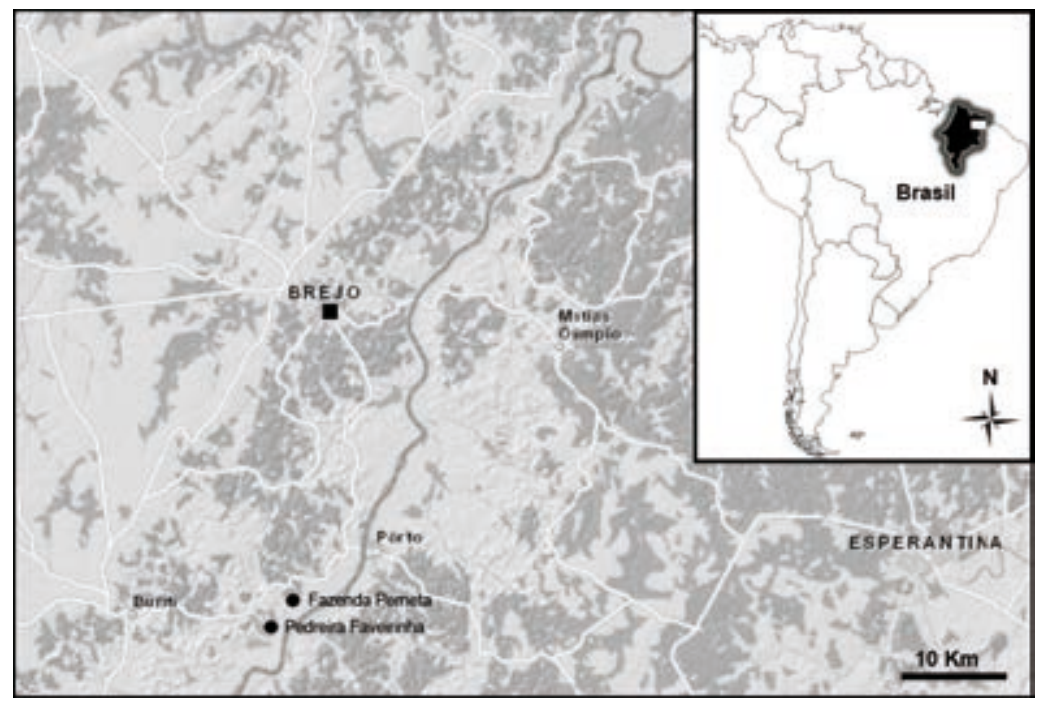

Fig. 1 - Mapa de localização dos principais afloramentos fossilíferos no município de Brejo, Estado do Maranhão (Fazenda Perneta e Pedreira Faveirinha).

\section{2 - Contexto geológico}

Os crustáceos, aqui analisados, provêm da bacia intracratônica do Parnaíba, situada numa ampla área do nordeste ocidental brasileiro, abrangendo os estados do Maranhão, Piauí e parte do Tocantins, Pará, Ceará e Goiás. Esta bacia possui uma área total de $600.000 \mathrm{~km}^{2}$ e sua sucessão sedimentar, em seu depocentro, registra $3.500 \mathrm{~m}$ de espessura, das quais $500 \mathrm{~m}$ pertencem a rochas mesozoicas e $2.500 \mathrm{~m}$ são de idade paleozoica (CAMPBELL, 1949; MESNER \& WOOLDRIDGE, 1964).

A sucessão sedimentar e magmática da Bacia do Parnaíba é dividida em cinco supersequências: Siluriana, Mesodevoniana, Eocarbonífera, Neocarbonífera-Eotriássica, Jurássica e Cretácea, sendo esta última constituída pelas seguintes formaçóes: Corda, Grajaú, Codó e Itapecuru (VAZ et al., 2007). No entanto, segundo CARNEIRO (1974) e REZENDE (2002), as formações Corda, Grajaú e Codó são interdigitadas e equivalentes cronoestratigraficamente. A Formação Corda é composta por arenitos castanho-avermelhados muito finos e semifriáveis a semicoesos. A Formação Grajaú, por sua vez, compôe-se de arenitos creme-claro-esbranquiçados médio/grossos e níveis conglomeráticos. Na Formação Codó ocorrem folhelhos betuminosos, siltitos, calcários, evaporitos e arenitos. Sobreposta a estes depósitos ocorre a Formação Itapecuru, de idade mesoalbiana-neocretácea, composta por arenitos variegados, finos, friáveis com estruturas diversas e pelitos (VAZ et al., 2007).

As áreas de ocorrência da Formação Codó são restritas e descontínuas, e aparecem no leito dos rios que drenam o centro da bacia, desde a margem oeste, na confluência dos rios Tocantins e Araguaia, até próximo à margem do rio Parnaíba, na cidade de Brejo (SANTOS \& CARVALHO, 2009). A idade inferida para estes depósitos é aptiana/ 
albiana (LIMA, 1982) e seus estratos depositados em regime climático árido a semi-árido (ROSSETTI et al., 2001).

Segundo LIMA \& LEITE (1978), os registros paleontológicos evidenciam sedimentação em ambientes marinhos e salobro-lacustrinos. ROSSETTI et al., (2001), em análise estratigráfica e faciológica na região de Codó, Estado do Maranhão, interpretou três associaçóes de fácies: (1) lago central; (2) lago transicional; (3) lago marginal. À sequência superior foram interpretados ambientes deposicionais correspondentes a shoreface superior, laguna/baía interdistributária, lobos de suspensão e canal distributário (ROSSETTI et al., 2001; PAZ \& ROSSETTI, 2001). Em estudo palinoestratigráfico da Formação Codó, ANTONIOLI (2001) a dividiu em três unidades litoestratigráficas: (1) Inferior, apresentando caráter marinho incipiente; (2) Média, essencialmente evaporítica; e (3) Superior, com caráter marinho preclaro.

\section{3 - Material e métodos}

O material de estudo foi coletado em dois sítios fossilíferos localizados a cerca de 20 km da cidade de Brejo (Fazenda Perneta e Pedreira Faveirinha; Fig. 2), Estado do Maranhão. Os espécimes encontram-se depositados na coleção paleontológica da Universidade Federal do Rio de Janeiro (UFRJ-DG) sob os códigos (UFRJ-DG 156 Cr; UFRJ-DG 160 Cr; UFRJ-DG 168 Cr; UFRJ-DG 170 Cr). O material foi submetido a técnicas mecânicas de preparação e, em seguida, catalogados e fotografados para estudo.
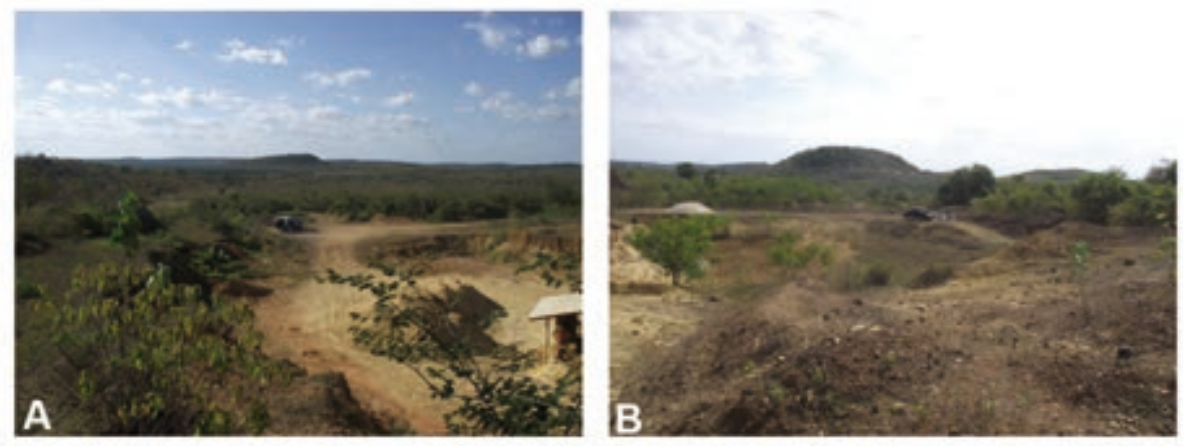

Fig. 2 - Afloramentos fossilíferos no município de Brejo, Estado do Maranhão.

(A) Fazenda Perneta; (B) Pedreira Faveirinha.

\section{4 - Resultados}

Decápodes ocorrem fosfatizados em calcários maciços e laminados, podendo variar de 1 a $3 \mathrm{~cm}$ de comprimento. O maior deles, UFRJ-DG $156 \mathrm{Cr}$, exibe um cefalotórax amplo e um abdômen levemente comprimido dorsoventralmente em relação ao cefalotórax. Apêndices cefálicos estão parcialmente preservados, enquanto pereiópodes, pleópodes e 
somitos encontram-se ausentes (Fig. 3 A). Outro espécime, UFRJ-DG 168 Cr, exibe apenas três pereiópodes preservados com quelas (Fig. 3 B). Entre os decápodes, ocorre ainda uma rara impressão de apêndice, possivelmente relacionada à infra-ordem Brachyura, UFRJ-DG $160 \mathrm{Cr}$ (Fig. 3 C). Contudo, a falta de caracteres diagnósticos, devido aos aspectos preservacionais, impossibilita uma determinação taxonômica efetiva. O espécimen UFRJ-DG $170 \mathrm{Cr}$ constitui um isópode com $15 \mathrm{~mm}$ de comprimento e exibe uma cabeça sub-retangular, profundamente inserida no primeiro pereionite; um dos olhos preservados está situado dorsolateralmente. Possui cerca de 10 pereionites similares com expansóes pleurais laterais arqueados distalmente; o pleotélson possui forma subtriangular. Dado o conjunto de características supracitadas, UFRJ-DG $170 \mathrm{Cr}$ constitui novo gênero e espécie de isópode Archaeoniscidae para a Formação Codó (Fig. 3 D).

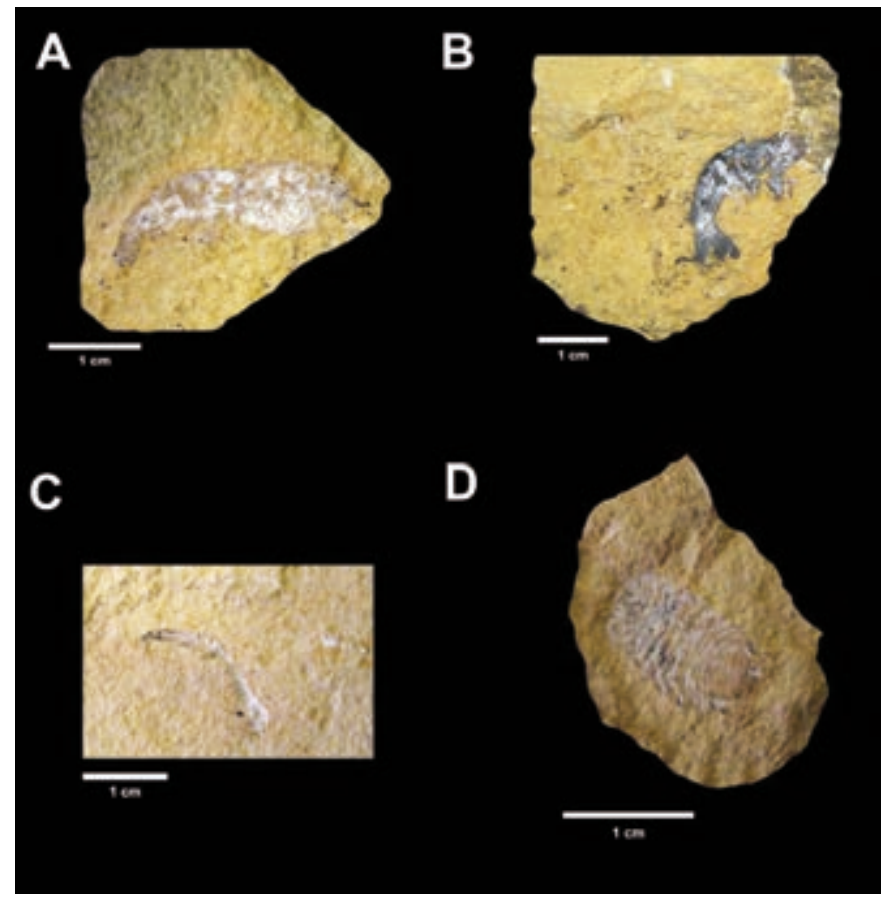

Fig. 3 - Crustáceos da Formação Codó. A, B. decápodes indet.

(UFRJ-DG 156 Cr, UFRJ-DG 168 Cr); C. impressão de apêndice de Brachyura

(UFRJ-DG 160 Cr); D. isópode Archaeoniscidae (UFRJ-DG $170 \mathrm{Cr}$ ).

\section{5 - Consideraçóes finais}

Crustáceos da Formação Codó eram conhecidos apenas por conchostráceos e ostracodes. Trabalhos de campo realizados no município de Brejo, Maranháo, possibilitaram a descoberta de novos taxa para esta unidade, o que sugere uma diversidade maior do que se supunha. Tais achados têm corroborado inferências paleoambientais anteriores para a 
Formação Codó (lagunar com breves incursões marinhas), como sugere o novo gênero e espécie de isópode Archaeoniscidae, típico de ambiente marinho. Decápodes (camarôes e caranguejos) constituem fósseis raros e eram desconhecidos para esta unidade. Contudo, a falta de caracteres diagnósticos, devido aos aspectos preservacionais, impossibilita uma determinação taxonômica detalhada. Assim, a coleta de novos espécimes em bom estado preservacional se faz necessária para um melhor entendimento da diversidade das biotas aquáticas durante a abertura do Atlântico Sul.

Agradecimentos - Os autores agradecem à Fundação de Amparo à Pesquisa e ao Desenvolvimento Científico e Tecnológico do Maranhão (FAPEMA), à Coordenação de Aperfeiçoamento de Pessoal de Nível Superior (CAPES), à Fundação de Amparo à Pesquisa do Rio de Janeiro (FAPERJ), Centro de Pesquisa de História Natural e Arqueologia do Maranhão (CPHNAMA) e ao Conselho Nacional de Desenvolvimento Científico e Tecnológico (CNPq) pelo apoio financeiro ao desenvolvimento deste estudo.

\section{Referências Bibliográficas}

ANTONIOLI, L. (2001) - Estudo palino-cronoestratigráfico da Formação Codó - Cretáceo Inferior do Nordeste brasileiro. Programa de Pós-Graduação em Geologia, Universidade Federal do Rio de Janeiro, Tese de Doutorado, $265 \mathrm{p}$.

BEURLEN, K. (1950) - Alguns restos de crustáceos decápodos d'água doce fósseis no Brasil. Anais da Academia Brasileira de Ciências, Rio de Janeiro, 22, p. 453-459.

BEURLEN, K. (1958) - Dois crustáceos do Cretáceo Superior do Nordeste do Brasil (Decapoda, Brachyura). Boletim do Museu Nacional, Nova Série Geológica, Rio de Janeiro, Brasil, 26, p. 1-23.

CAMPBELL, D. F. (1949) - Revised report on the reconnaissance geology of the Maranhão Basin. Conselho Nacional do Petróleo, Rio de Janeiro, 117p. Relatório Interno.

CARDOSO, R. N. (1962) - Alguns conchostráceos mesozoicos do Brasil. Boletim da Sociedade Brasileira de Geociências, 25, p. 21-28.

CARNEIRO, M. V. (1974) - Mapeamento estrutural da área de Grajaú-Imperatriz. Petrobras, Relatório Técnico, 358, Belém, 45 p.

DUARTE, L. \& SANTOS, R. S. S. (1962) - Fósseis do Arenito Açu. Anais da Academia Brasileira de Ciências, 34 I, p. 57-68.

KRÖMMELBEIN, K. \& WEBER, R. (1985) - Ostracodes do "Wealden” do Nordeste brasileiro. Tradução. G. Beurlen. Rio de Janeiro, Petrobras, Cenpes, Sintep, Ciência-Técnica-Petróleo, Seção de Exploração de Petróleo, Publ. 16, 63p.

LEITE, J. F., ABOARRAGE, A. M. \& DAEMON, R. F. (1975) - Projeto carvão da Bacia do Parnaíba. Relatório Final das Etapas II e III, DNPM/CPRM, Recife, $5 \mathrm{v}$.

LIMA, M. R. (1982) - Palinologia da Formação Codó na regiāo de Codó, Maranhão. Boletim do Instituto de Geociências, USP, 13, p. 116-128.

LIMA, E. A. A. \& LEITE, J. F. (1978) - Projeto estudo global dos recursos minerais da bacia sedimentar do Parnaíba. Recife, Integração Geológica-Metalogenética, DNPM/CPRM, 437p. Relatório.

LiNDOSO, R. M., CARVAlHO, I. S., MEDEIROS, M. A., PEREIRA, A. A., SANTOS, R. A. B., MENDES, I. D., BRITO, J. M., VILAS BÔAS, I., ARAÚJO, M. N. \& FERREIRA, N. N. (2011) - Novos sítios fossilíferos em carbonatos da Formação Codó (Aptiano/Albiano) da Bacia do Parnaíba, Maranhâo, Brasil. 
In: Carvalho, I. S., Srivasatava, N. K., Strohschoen Jr, O. S. \& Lana, C. C. (eds.). Paleontologia: Cenários de Vida. Vol. 4, Editora Interciência, p. 819-827.

MAISEY, J. G. \& CARVALHO, M. G. P. (1995) - First records of fossil Sergestid Decapods and fossil Brachyuran crab larvae (Arthropoda, Crustacea), with remarks on some supposed palaemonid fossils, from the Santana Formation (Aptian-Albian, NE Brazil). American Museum Novitates, New York, 3132, p. 1-7.

MARTINS-NETO, R. G. \& MEZZALIRA, S. (1991) - Descrição de novos crustáceos (Caridea) da Formação Santana, Cretáceo Inferior do Nordeste do Brasil. Anais da Academia Brasileira de Ciências, 63, p. 362-367.

MAURY, C. J. (1930) - O Cretáceo da Parahyba do Norte. Monografia do Serviço Geológico e Mineralógico do Brasil. Anais da Academia Brasileira de Ciências, 63, p. 155-160.

MESNER, J. C. \& WOOLDRIDGE, L. C. P. (1964) - Estratigrafia das bacias paleozoica e cretácea do Maranhão. Rio de Janeiro, Boletim Técnico Petrobras 7, p. 137-164.

PAZ, J. D. S. \& ROSSETTI, D. F. (2001) - Reconstrução paleoambiental da Formação Codó (Aptiano), borda leste da Bacia do Grajaú, MA. In: Rossetti, D. F.; Góes, A. M. e Truckenbrodt, W. (eds.). O Cretáceo na Bacia de São Luís-Grajaú. Belém, Museu Paraense Emílio Goeldi, Coleção Friedrich Katzer, p. 77-100.

REIS, M. A. F., TURBAY, C. V. G. \& CESERO, P. (2005) - Descrição de um novo Decapoda (Natantia, Malacostraca, Crustacea) da Formação Riachuelo, Albiano da Bacia de Sergipe. Anuário do Instituto de Geociências-UFRJ, 28, p. 80-91.

REZENDE, N. G. A. M. (2002) - A zona zeolítica da formação corda, Bacia do Parnaíba. 2002. Dissertação (Mestrado) - Universidade Federal do Pará, Belém, 142 p.

ROSSETTI, D. F., GÓES, A. M. \& ARAI, M. (2001) - A passagem Aptiano-Albiano na Bacia do Grajaú. In: Rossetti, D. F., Góes, A. M. \& Truckenbrodt, W. (eds.). O Cretáceo na Bacia de São Luís-Grajaú. Belém, Museu Paraense Emílio Goeldi, Coleção Friedrich Katzer, p. 101-117.

ROXO, M. G. O. (1940) - Preliminary note on fóssil crustacea from Bahia, Brazil. Anais da Academia Brasileira de Ciências, 22, p. 279-280.

SANTOS, M. E. C. M. (1971) - Um novo artrópodo da Formação Areado, Estado de Minas Gerais. Anais da Academia Brasileira de Ciências, 43, p. 415-420.

SANTOS, M. E. C. M. \& CARVALHO, M. S. S. (2009) - Paleontologia das bacias do Parnaíba, Grajaú e São Luís. Rio de Janeiro: CPRM Serviço Geológico do Brasil - DGM/DIPALE, 215 p.

SCHWEIGERT, G., MARTILL, D. M. \& WILLIAMS, M. (2007) - Crustacea of the Crato Formation. The Crato Fossil Beds of Brazil. In: Martill, D. M., Bechly, G. e Loveridge, R. F. (eds). Cambridge University Press, p. 133-141.

SILVA, M. D., KAERCHER, E. G. \& BARBOSA, E. G. S. (1985) - Bioestratigrafia do furo 1-UN-32-PI, Roça do Meio, Municipio de Duque Bacelar, Formação Codó, Cretáceo Inferior, bacia do Maranhão. In: Congresso Brasileiro de Paleontologia 9, 1985, Fortaleza, Resumos..., Fortaleza, SBP, 80 p.

SILVA, M. D., BARBOSA, E. G. S. \& KAERCHER, E. G. (1989) - Bioestratigrafia do furo 1-UN-24, Buriti, Maranhão, Formação Codó, Cretáceo Inferior da bacia do Maranhão. In: Simpósio de Geologia do Nordeste 13, 1989, Fortaleza, Atas..., 1989, SBG, Núcleo Nordeste, p. 188-192.

TÁVORA, V. A. \& SOUZA-LIMA, W. (2001) - Os fósseis da Bacia de Sergipe-Alagoas. www.phoenix.org. br/Phoenix27_mar01 (consultado em: 2011.05.14)

VAZ, P. T., REZENDE, N. G. A. M., FILHO, J. R. W. \& TRAVASSOS, W. A. S. (2007) - Bacia do Parnaíba. Boletim de Geociências Petrobras, Rio de Janeiro, 15, p. 253-263. 